\title{
DEMOSAICING LOW RESOLUTION QVGA BAYER PATTERN
}

\author{
First Author Name, Second Author Name \\ Institute of Problem Solving, XYZ University, My Street, MyTown, MyCountry \\ f_author@ips.xyz.edu,s_author@ips.xyz.edu \\ Third Author Name \\ Department of Computing, Main University, MySecondTown, MyCountry \\ t_author@xy.mu.edu
}

Keywords: $\quad$ robot vision, demosaicing, QVGA resolution, Bayer pattern

\begin{abstract}
In this paper, we present a solution for the interpolation of low resolution digital images. Many digital cameras can function in two resolution modes: VGA (i.e., $640 \times 480$ ) and QVGA (i.e., $320 \times 240$ ). These cameras use a single sensor covered with a Color Filter Array (CFA). The CFA allows only one color component to be measured at each pixel, the remaining color components must be interpolated, this operation is called demosaicing. There is not a standard way to interpolate the QVGA Bayer pattern and most of the known demosaicing algorithms are not suitable. In this paper, we propose a new solution for the interpolation of QVGA Bayer pattern. Experimental results using digital images and an evaluation function confirm the effectiveness of the interpolation method.

The use of the QVGA resolution is important in low-cost and low-power embedded hardware. As an application, we chose the RoboCup domain and in particular our Robovie-M humanoid robot competing in the RoboCup Kid-Size Humanoids League.
\end{abstract}

\section{INTRODUCTION}

Color digital cameras are popular today. They can be found also in PDA cellular phone, etc. CMOS digital cameras are more and more often used in autonomous robots to acquire images of the environment surrounding a robot.

High-quality digital color cameras use three-chip sensor, to acquire images. The light entering the camera is split and projected onto a different sensor for each spectral component. Each sensor has to be aligned properly and requires its own electronics. These additional requirements made three-chip sensor cameras very expensive.

To avoid these costs, many cameras use a single sensor covered with a Color Filter Array (CFA). The CFA is a colored filter that allows only one color component to be measured at each pixel, the remaining color components must be interpolated: this operation is called demosaicing. Several patterns exist for the CFA, the most common is the Bayer pattern, shown in Fig. 1(a).

Many algorithms for demosaicing the Bayer pat- tern exist in literature. In this paper we focus on low computational cost algorithm that are intended for embedded low power systems.

Low cost digital cameras with CFA can acquire images at different resolutions: the most important are high resolution VGA and low resolution QVGA. In low resolution acquisition mode, the Bayer pattern is sampled to reduce the data rate. This reduction is obtained skipping two consecutive columns every four, as shown in Fig. 1(b), obtaining what we call QVGA Bayer pattern. In this paper, we propose a new solution for the interpolation of QVGA Bayer pattern, obtained sampling only columns, based on periodic reconstruction.

The QVGA resolution is adopted in many devices such as cellular phones and PDAs with inexpensive cameras. The QVGA interpolation algorithm proposed in this paper could also be used to improve the color image acquisition in these handheld devices or for image visualization in small LCDs.

We use QVGA low resolution mode in our KidSize Humanoid to reduce the data rate and to improve image processing speed, maintaining a large field of 


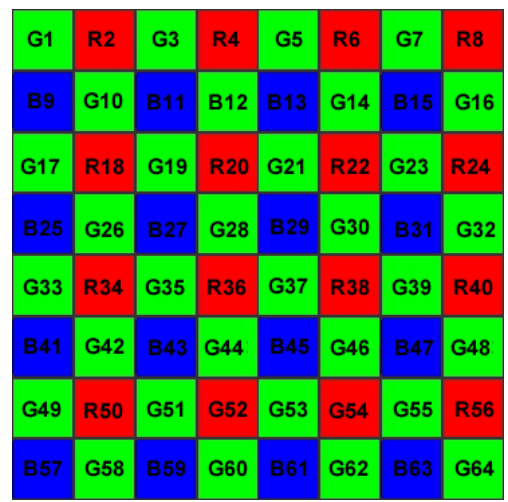

(a) VGA mode.

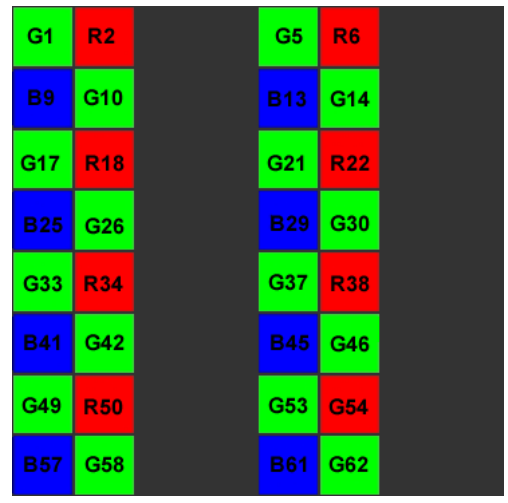

(b) QVGA mode.

Figure 1: Bayer pattern base structure VGA and QVGA mode.

view and reducing the resolution. We use QVGA resolution for full field of view and VGA for focalized view reduced to a region of interest of the image, where an object of interest has been detected.

We also introduce an evaluation function to compare the different interpolation methods for QVGA; this function shows effectiveness of improvement of quality in interpolation results due to periodic reconstruction.

The remainder of the paper is organized as follows. In Section 2, we summarize the most common interpolation methods for the Bayer pattern. In Section 3, we present basic interpolation methods for QVGA Bayer pattern and the new one proposed by us. In Section 4 these algorithm are compared using quality evaluation functions. In Section 5 we propose an application of new algorithm for focus of attention humanoid robots.

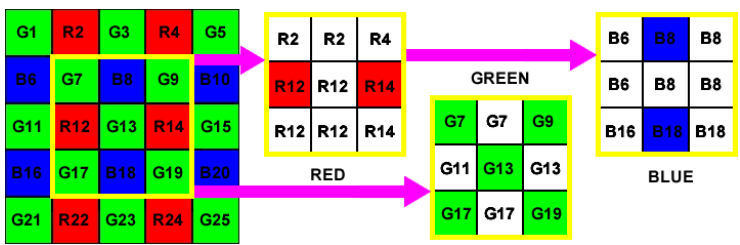

Figure 2: Nearest Neighbor Replication in VGA Bayer pattern.

\section{DEMOSAICING BAYER PATTERN}

Consider a $2 \times 2$ base grid, the Bayer pattern measures the $\mathrm{G}$ in two diagonal pixel and the $\mathrm{R}$ and $\mathrm{B}$ in the others, as shown in Fig. 1(a). The G component is measured twice with respect to $\mathrm{R}$ or $\mathrm{B}$ because the peak sensitivity of the human visual system lies in the medium wavelengths, corresponding to the $\mathrm{G}$ portion of the visible spectrum. The computer vision community has devoted many efforts to the problem of Bayer pattern demosaicing. There are many algorithms to interpolate the Bayer pattern. In this paper, we consider only low computational algorithm. For a comprehensive description of the current state of the art see the work of Gunturk et al. (Gunturk et al. (2005)).

The Nearest Neighbor Replication method is the simplest algorithm for demosaicing CFA images. It is analyzed in the works of (Parulski (1985)), (Adams (1995)), (Adams and Hamilton (1997)), (Zen et al. (1998)) and of (Sakamoto et al. (1998)).

The algorithm is non-adaptive, i.e. operates in the same way for all pixel of the same components in every images. The value of each interpolated pixel is obtained by copying the value of the nearest pixel in the Bayer pattern image, as shown in Fig. 2. The nearest pixel can be any one of the pixel in the neighborhood: upper, lower, left and right pixels.

This algorithm does not involve any arithmetic operation and therefore this method does not impose a heavy computational cost. However there are many negative aspects: creation of false colors and high chromatic gradient transitions become irregular, this effect is called zipper effect.

The Bilinear Interpolation is the most used algorithm for demosaicing CFA images. It is analyzed in the works of (Parulski (1985)), (Wu et al. (1997)), (Chan et al., 1996), (Tsai et al. (1997)), (Zen et al. (1998)), (Adams et al. (1998)) and also of (Sakamoto et al. (1998)). In other works, such as in (Lukin (2004a)) and in (Lukin (2004b)) Bilinear Interpolation method forms the base for more sophisticated 


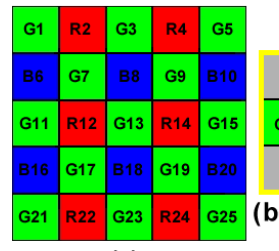

(a) (c)

(d)

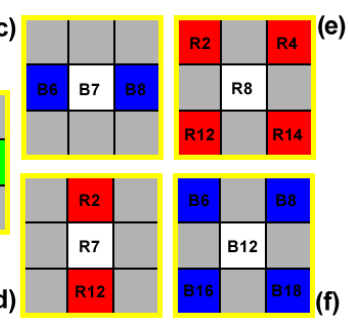

Figure 3: Bilinear Interpolation in VGA Bayer pattern. algorithm; in (Malvar (2004)) this method is studied for non-linear interpolation algorithm on rhomboidal grid.

The algorithm is non-adaptive. There are two privileged directions (i.e., vertical and horizontal) and pixels are bilinearly interpolated along these directions, as shown in Fig. 3.

An interpolation algorithm that has good performances in terms of quality of reconstruction is Linear Interpolation with Laplacian Second-order Correction Terms that we adopted in our application. The Linear Interpolation with Laplacian Second-order Correction Terms is an adaptive algorithm, i.e. it changes the interpolation equation adapting to the image to be interpolated. This algorithm is analyzed in (Hamilton and Adams (1997)) and with modifications in (Adams and Hamilton (1997)) and (Adams et al. (1997)).

Interpolation of the green component has to be computed before the other components. Consider interpolation of green component in blue pixel, see Fig. 4(a), for example $G 5$ in B5. Calculate gradient in horizontal $(\Delta H)$, and vertical $(\Delta V)$ direction

$$
\begin{aligned}
\Delta H & =|G 4-G 6|+|B 5-B 3+B 5-B 7| ; \\
\Delta V & =|G 2-G 8|+|B 5-B 1+B 5-B 9| .
\end{aligned}
$$

Then evaluate which interpolation fuction has to be used:

$$
\begin{aligned}
\Delta H<\Delta V & \rightarrow \quad G 5=\frac{(G 4+G 6)}{2}+\frac{(2 \cdot B 5-B 3-B 7)}{4} \\
\Delta H>\Delta V & \rightarrow \quad G 5=\frac{(G 2+G 8)}{2}+\frac{(2 \cdot B 5-B 1-B 9)}{4} \\
\Delta H=\Delta V & \rightarrow \quad G 5=\frac{(G 2+G 4+G 6+G 8)}{4}+ \\
& +\frac{(4 \cdot B 5-B 1-B 3-B 7-B 9)}{8}
\end{aligned}
$$

For red and blue components are possible tree cases, consider Fig. 4(b):

1. interpolate red or blue component in green position when nearest same color components are in (a)
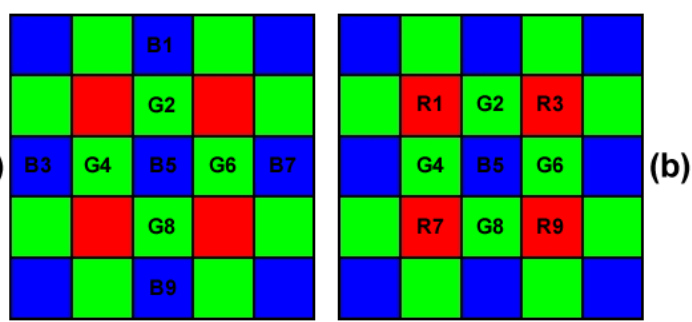

Figure 4: Linear Interpolation with Laplacian Second-order Correction Terms in VGA Bayer pattern.

the same column. Consider $R 4$ in $G 4$, interpolation is done with (7)

$$
R 4=\frac{(R 1+R 7)}{2}+\frac{(G 4-G 1+G 4-G 7)}{4} ;
$$

2. interpolate red or blue component in green position when nearest same color components are in the same row. Consider $R 2$ in $G 2$, interpolation is done with (8)

$$
R 2=\frac{(R 1+R 3)}{2}+\frac{(G 2-G 1+G 2-G 3)}{4} ;
$$

3. interpolate red or blue component in blue or red position. Consider $R 5$ in B5. Define diagonal gradient in negative direction $\Delta N(9)$ and in positive direction $\Delta P(10)$.

$$
\begin{aligned}
\Delta \mathrm{N} & =|R 1-R 9|+|G 5-G 1+G 5-G 9|(9) \\
\Delta P & =|R 3-R 7|+|G 5-G 3+G 5-G 7|(10)
\end{aligned}
$$

Then evaluate which interpolation fuction has to be used:

$$
\begin{aligned}
\Delta \mathrm{N}<\Delta P & \left.\rightarrow R 5=\frac{(R 1+R 9)}{2}+\frac{(2 \cdot G 5-G 1-G 9)}{4}\right) \\
\Delta \mathrm{N}>\Delta P & \rightarrow \quad R 5=\frac{(R 3+R 7)}{2}+\frac{(2 \cdot G 5-G 3-G 7)}{4} \\
\Delta \mathrm{N}=\Delta P & \rightarrow \quad R 5=\frac{(R 1+R 3+R 7+R 9)}{4}+ \\
& +\frac{(4 \cdot G 5-G 1-G 3-G 7-G 9)}{8}
\end{aligned}
$$

\section{DEMOSAICING QVGA BAYER PATTERN}

As we already said in the introduction, the QVGA Bayer pattern is obtained sampling the Bayer pattern skipping two columns every four, see Fig. 1(b). To the best of our knowledge, there is not a standard way in literature to interpolate the QVGA Bayer pattern. In this paper we propose a new interpolation method. 


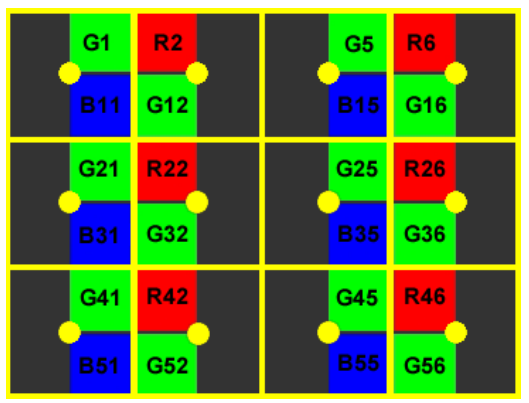

(a) QVGA structure.

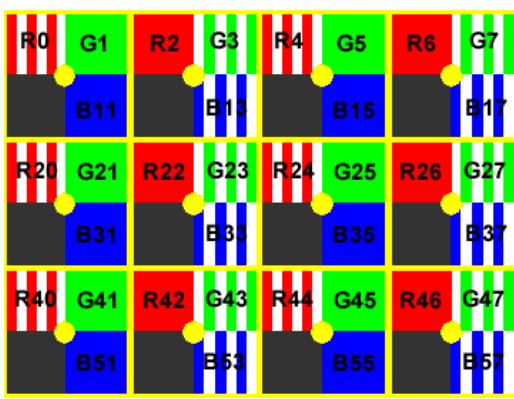

(b) QVGA periodic reconstruction.

Figure 5: Base structure of QVGA and periodic reconstruction.

The main idea originates from an original work of (Tang and Lee (2004)) about interpolation of classical Bayer pattern. In that work, Tang and Lee proposed a vertex based interpolation method instead of center based interpolation method.

Similarly, we based our interpolation algorithm for QVGA on vertex interpolation mode (i.e. the interpolated pixels are centered on the vertex of the Bayer pixels). This is sketched in Fig. 5(a). The interpolated pixel is the yellow square binding $2 \times 2$ Bayer pixels. The yellow dot represents the center of the interpolated pixel. With respect to the VGA Bayer pattern, the QVGA Bayer pattern has the following peculiarities:

1. in each yellow square one color component is missing;

2. the vertical periodicity of the grid is of 2 pixels, while the horizontal is of 4 pixels;

3 . in the yellow squares along the same column is missing always the same color component;

In the next paragraphs, we will show how the Nearest Neighbor Replication algorithm and the Bilinear Interpolation algorithm could be applied to the QVGA Bayer pattern. We will see that both algorithms perform poorly, basically because they do not use a periodic pattern for the color components. In

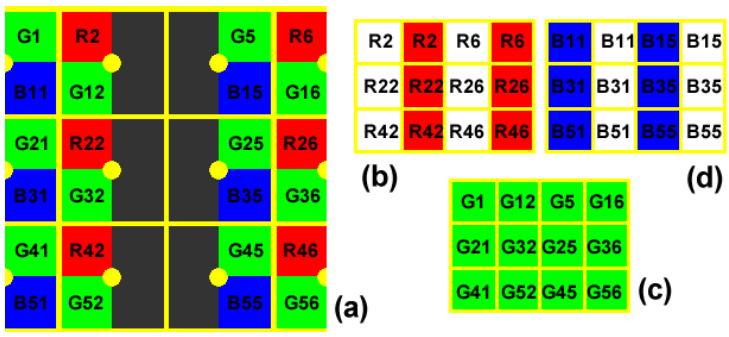

Figure 6: Nearest Neighbor Replication in QVGA Bayer pattern.

Section 3.3, we propose a new method called Periodic Reconstruction Interpolation in which some of the color components are interpolated to obtain a periodic color pattern, that is equally repeated in every yellow square of the QVGA image, as depicted in Fig. 5(b). Interpolated pixels are always painted with the corresponding color and white bar pattern.

\subsection{Nearest Neighbor Replication}

The Nearest Neighbor Replication algorithm is the simplest non-adaptive algorithm for demosaicing QVGA images. The green component does not need to be interpolated; every yellow square contains a green pixel of the QVGA Bayer pattern. The red and the blue pixels are obtained by replicating, respectively, the nearest right pixel and the left nearest pixel, as shown in Fig. 6. The pros and cons of this method are the same as the algorithm presented in VGA mode: low computational cost, presence of false color and zipper effect.

\subsection{Bilinear Interpolation}

The Bilinear Interpolation algorithm is a nonadaptive algorithm for demosaicing QVGA images, that makes an average on vertical and horizontal direction.

The green component, as in Nearest Neighbor Replication algorithm, is not interpolated. For the interpolation of red and blue components, see Fig. 7, is not possible to have an exactly bilinear interpolation, because the color information in vertical direction is always absent.

The interpolation method uses a weighted mean based on the distance. For a red pixel, for example $R 24$, the value is obtained with (15)

$R 24=\frac{(4 \cdot R 22+4 \cdot R 26+3 \cdot R 2+3 \cdot R 6+3 \cdot R 42+3 \cdot R 46)}{20}$, 


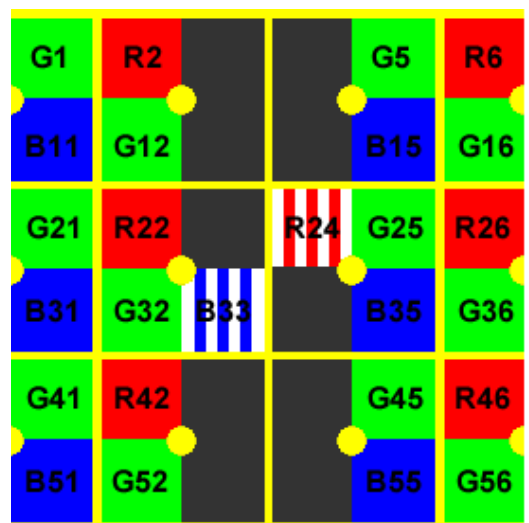

Figure 7: Bilinear interpolation in QVGA Bayer pattern.

and for a blu pixel, as $B 33$, consider Eq. (16)

$B 33=\frac{(4 \cdot B 31+4 \cdot B 35+3 \cdot B 11+3 \cdot B 15+3 \cdot B 51+3 \cdot B 55)}{20}$.

Unfortunately this algorithm blurs the image by averaging over all pixels without any appropriate weighting. Even this algorithm is affected by the zipper effect.

\subsection{Periodic Reconstruction Interpolation}

A thorough analysis of the Nearest Neighbor Replication and Bilinear Interpolation algorithms when used for the interpolation of QVGA Bayer pattern shows that they do not correctly interpolate the green component. This problem arises from the non-periodic interpolation domain (i.e., the distance between two green pixels varies along the grid). This results in a wrong attribution to green components. We propose first to interpolate the green components to construct the periodic grid of Fig. 5(b), from this the name Periodic Reconstruction Interpolation, and then to interpolate the red and blue components.

\subsubsection{Interpolation of Green Pixel Components.}

The green component in every yellow square is obtained as the average of the nearest green Bayer pixels weighted by their distances. As an example consider pixel G23, as shown in Figure 8(a). The interpolation is obtained by (17)

$$
G 23=\frac{(3 \cdot G 21+4 \cdot G 12+4 \cdot G 32+3 \cdot G 25)}{14} .
$$

To limit the low-pass filter effect due to the average operation, we perform it only on pixel closer than two pixels from the interpolation center.

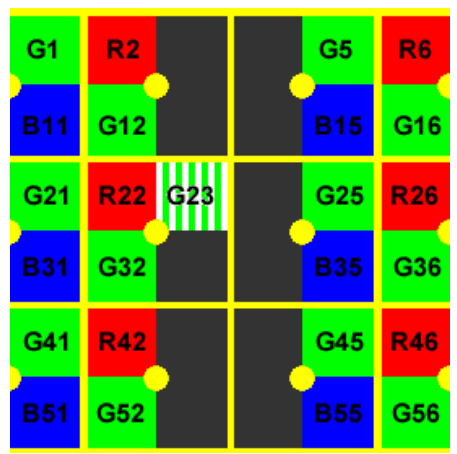

(a) Green.

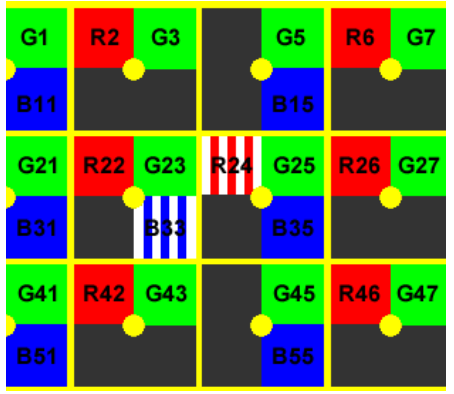

(b) Red and Blue.

Figure 8: Periodic Reconstruction Intepolation in QVGA Bayer pattern.

\subsubsection{Interpolation of Red and Blue Pixel Components.}

The next step is to create a periodic structure also for these components. Consider Fig. 8(b) where green pixels have been already interpolated by the Eq. (17). We propose an adaptive algorithm for interpolation of red and blue pixels.

Consider red pixel $R 24$. We define diagonal gradients in negative direction as $\Delta N(18)$ and in positive direction as $\Delta P(19)$ and the horizontal gradient as $\Delta O$ (20).

$$
\begin{aligned}
\Delta N & =|R 2-R 46|+|G 25-G 3+G 25-G 47| ;(18) \\
\Delta P & =|R 6-R 42|+|G 25-G 7+G 5-G 43| ;(19) \\
\Delta O & =|R 22-R 26|+\mid G 25-G 23+G 25-G 27(20)
\end{aligned}
$$

The value of every interpolated pixel is calculate with Algo 3.1 depending on which of the gradient is maximum. The interpolation of blue pixel is done in the same way. 


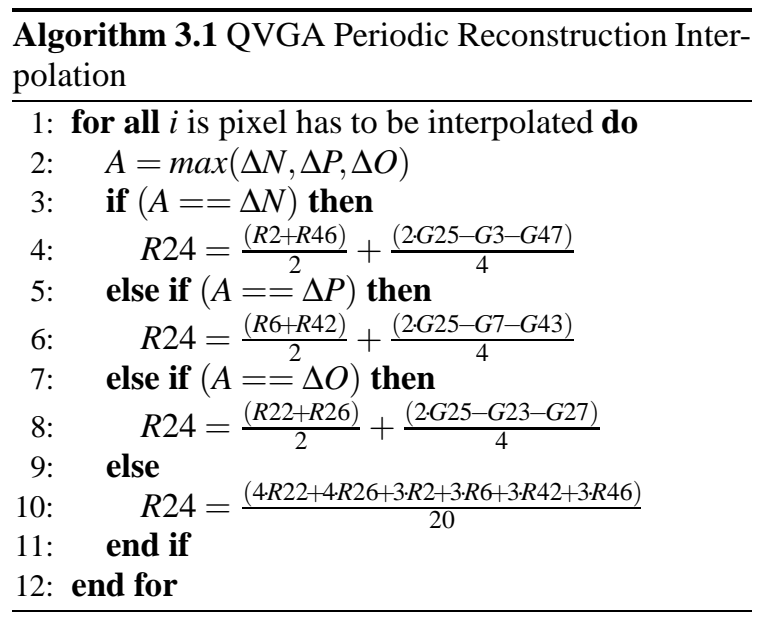

\section{ALGORITHM QUALITY EVALUATION}

To compare the quality of the images reconstruction with different algorithms, in addition to the visual comparison of the reconstructed images, we use the dependency index developed in (Deleted (2006)). This index is based on the concept of entropy, see (Deleted (2006)).

The original and reconstructed images to be compared has to be considered as a linear array. In bivariate case the dependency index ${ }_{P \mid F} \tilde{D}$ is defined as (21), where $P$ represent the pixel's position in the linear array and $F$ indicates the original and reconstructed image. ${ }_{F} H$ and ${ }_{P} H$ is denoted the marginal entropy respect figure (F) and position $(\mathrm{P})$, with ${ }_{P F} H$ the joined entropy and with ${ }_{P F} I$ the information between original and reconstructed figure.

$$
{ }_{P \mid F} \tilde{D}=\frac{{ }_{P F} I}{{ }_{P} H}=\frac{{ }_{F} H+{ }_{P} H-{ }_{P F} H}{{ }_{P} H} .
$$

In the trivariate case, where third qualitative variable is color (C) we consider the partial dependency index without effect of color (22)

${ }_{P \mid F} \tilde{D}_{C}=\frac{P \mid C H-{ }_{P \mid F C} H}{P \mid C}=\frac{P C H-{ }_{C} H-{ }_{P F C} H+{ }_{F C} H}{{ }_{P C} H-{ }_{C} H}$.

The dependency indexes are for construction normalized in the range $[0,1]$. If two images are very similar the dependency index tends to 0 .

We use dependency indexes ${ }_{P \mid F} \tilde{D}_{(R)},{ }_{P \mid F} \tilde{D}_{(G)}$ and ${ }_{P \mid F} \tilde{D}_{(B)}$ for bivariate case for each single components and ${ }_{P \mid F} \tilde{D}_{(T)}$ for image in all components. We use partial dependency index without effect of color ${ }_{P \mid F} \tilde{D}_{C}$ for trivariate case.
To assess to interpolation quality of different algorithm proposed, consider the Tab. 1 the which are listed dependency indexes. Periodic Reconstruction has ${ }_{P \mid F} \tilde{D}_{(G)}$ index that is half than other methods. This represent a good improvement in interpolation quality. In Fig. 9 interpolation algorithms have been visually compared. It is visible the presence of zipper effect an false colors in Fig. 9(b) and Fig. 9(c) corresponding to Nearest Neighbor Replication and Bilinear Interpolation alortithm. The Fig. 9(d) obtained with Periodic Reconstruction algorithm has sharp edges and no zipper effects.

\begin{tabular}{|c|c|c|c|c|c|}
\hline & \multicolumn{5}{|c|}{ Dependency index } \\
\hline & ${ }_{P \mid F} \tilde{D}_{(R)}$ & ${ }_{P \mid F} \tilde{D}_{(G)}$ & ${ }_{P \mid F} \tilde{D}_{(B)}$ & ${ }_{P \mid F} \tilde{D}_{(T)}$ & ${ }_{P \mid F} \tilde{D}_{C}$ \\
\hline$\overline{\text { Neighbor }}$ & 10.4765 & $\overline{5.8445}$ & $\overline{23.1138}$ & $\overline{10.2245}$ & $\overline{11.0308}$ \\
\hline Bilinear & 10.9063 & 5.8445 & 22.6590 & 10.2537 & 11.0618 \\
\hline Periodic & 7.3436 & 2.4420 & 15.2124 & 6.8667 & 7.5032 \\
\hline
\end{tabular}

Table 1: Comparison between interpolation methods on QVGA Bayer pattern, all values are in $10^{-5}$.

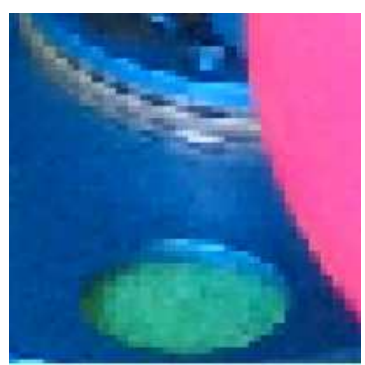

(a) Original

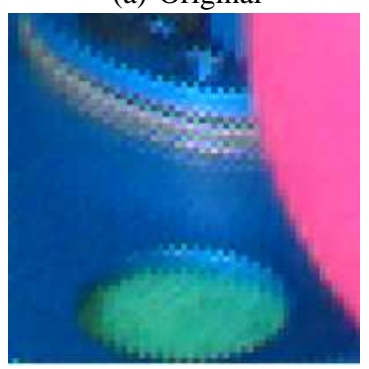

(c) Bilinear

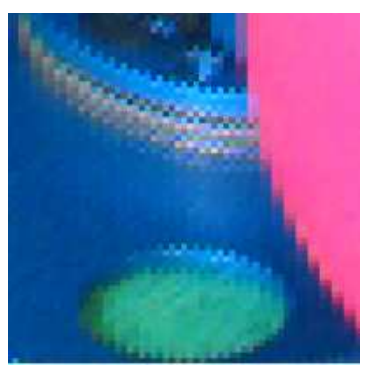

(b) Neighbor

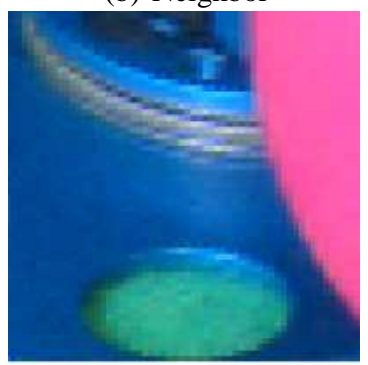

(d) Periodic
Figure 9: Visive comparison between QVGA interpolation methods.

\section{FOCUSING THE ATTENTION}

As said before, our robot can acquire images in two modes: low resolution (QVGA) and high resolution (VGA). Due to the hardware constraints of 
our humanoid robot, it is not possible to store a fullresolution image in memory. Storing a 640x480x 16 bit color image would require $614 \mathrm{~KB}$, while our robot has only $512 \mathrm{~KB}$ of working RAM. Therefore, we use low resolution to acquire a complete frame covering the whole field of view of the robot's camera, while we acquire at high resolution only the regions of interest (ROI) in which we want to focalize the attention into interesting objects in the scene (e.g., the ball or an opponent robot).

The platform on which this method is implemented is Robovie-M by VStone with a Renesas CPU at $40 \mathrm{Mhz}$ and a RAM of $256 \mathrm{k}-$ Word of $16-$ bit. The digital camera used is a CMOS camera (an OV3620 by Omnivision). We are planning to mount an omnidirectional mirror on the camera and so we acquire square images instead of the classical 4:3 images. This has the additional advantage of further reduce the memory space required by every image. Acquiring the $240 \times 240$ Bayer pattern with a QVGA resolution and interpolating it with the Periodic Reconstruction Interpolation, enables the robot to allocate memory space for three images to be used for image processing operations that cannot be done in place. With high resolution VGA (also $240 \times 240$ but with double density of pixels), interpolated with Linear Interpolation with Laplacian Second-order Correction Terms, the robot can focus the attention by zooming in a ROI corresponding to particular objects of interest, while having memory space always for three images. At the moment the attention mechanisms is rather trivial: The center of the high-resolution ROI is set on the center of mass of the blob of the object of interest (ball, opponent robot, goal). The current implementation of the image processing software is composed by image acquisition, interpolation, color segmentation and blob detection, we reached a performance of $2 \mathrm{fps}$ on a 40Mhz CPU that has also to control 22 motors during image elaboration time. An example of elaboration is shown in Fig. 10.

An improvement of focusing the attention module can be reached implementing an active sensor able to modify its own sensing function. Some cameras has a sensor that change its hardware configuration, like "attention-retina" of (Maris (2001)), or using the same camera with different algorithm. The visual attention sensor can not be designed separate from reasoning and motion control. On this idea are based works of (Reece e Shafer (1995)) to drive a autonomous vehicle in urban traffic or in (Okuno et al. (2004)) where visual and auditive sensation has to be merged. An example of humanoid robot with an active vision system, operating a real time visual attention was proposed in (Vijayakumar et al. (2001)). In

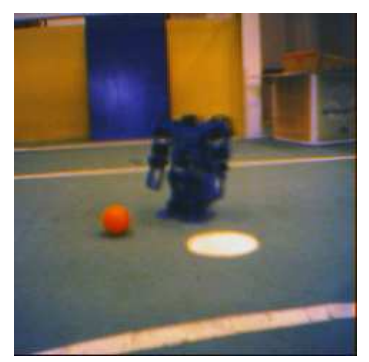

(a) QVGA panoramic

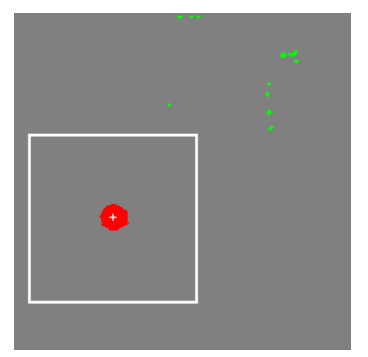

(b) Ball finder

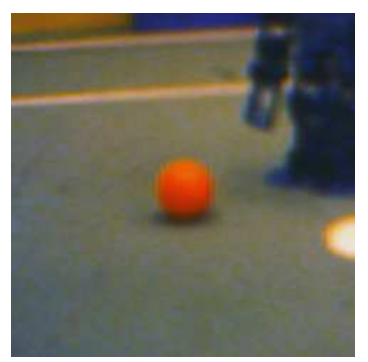

(c) VGA Zoomed

Figure 10: Focusing the attention image output.

that case attention is focalized over bright spot.

\section{CONCLUSION}

The use of QVGA format is important in low cost and low computational power systems as embedded PCs, PDAs and cellular phones. In this paper we present a new interpolation method for QVGA Bayer pattern: Periodic Reconstruction Interpolation. The improvement of quality of the images reconstructed with proposed method is demonstrated using dependency indexes.

We realize a simple focus of attention system in a humanoid platform using QVGA to have a large view of the environment around the robot and VGA resolution to focalize on particular features, like ball, and extract more accurate information. We showed the performances of $2 \mathrm{fps}$ obtained on an implementation in a 40Mhz CPU.

At the moment of writing, we are applying the proposed method to omnidirectional images and we are implementing more complex focus of attention system. We are evaluating an extension of Periodic Reconstruction Interpolation to other patterns, for example to pattern implemented in STMicroelectronics cameras, obtained skipping also two rows with the same ratio. 


\section{REFERENCES}

Adams, J.E.: Interactions between color plane interpolation and other image processing functions in electronic photography, Proceedings of SPIE 2416 (1995) 144-151.

Adams, J.E. and Hamilton, J.F.: Design of practical color filter array interpolation algorithms for digital cameras, Proceedings of SPIE 3028 (1997) $117-125$.

Adams, J.E. et al.: Adaptive color plane interpolation in single color electronic camera, U.S. Patent 5506619 (1997).

Adams, J.E., Parulski, K. and Spaulding, K.: Color Processing in Digital Cameras, IEEE Transactions on Microeletectronic, 186 (1998) 20-31.

Chan, W. et al.: A Mega-Pixel resolution PC Digital Still Camera, Proceedings of SPIE, 2654 (1996) 164-171.

Gunturk, B.K., Glotzbach, J., Altunbasak, Y., Schafer, R.W. and Mersereau, R.M.: Demosaicking: Color Filter Array Interpolation, exploring the imaging process and the correlations among three color planes in single-chip digital cameras, IEEE Signal Processing Magazine, 10 53 (2005) January 44-54.

Deleted for blind review: Deleted for blind review, deleted for blind review (2006).

Deleted for blind review: Deleted for blind review, deleted for blind review (2006).

Hamilton, J.F. and Adams, J.E.: Adaptive color plane interpolation in single sensor color electronic camera, U.S. Patent 5 629 734, (1997).

Lukin, A. and Kubasov, D.: An Improved Demosaicing Algorithm, Graphicon.2004 conference proceedings, Faculty of Applied Mathematics and Computer Science, State University of Moscow, Russia (2004).

Lukin, A. and Kubasov, D.: High-Quality Algorithm for Bayer Pattern Interpolation, Programming and Computer Software, 30 6 (2004) 347-358, translated from Programmirovanie, Original Russian Text, URL: http://audio.rightmark.org/lukin/graphics/resampling.htm/.

Malvar, H.S., He, L. and Cutler, R.: High-quality linear interpolation for demosaicing of Bayerpatterned color images, Microsoft Research One Microsoft Way, Redmond, WA, USA, (2004).

Maris, M.: Attention-based navigation in mobile robots using a reconfigurable sensor, Robotics and Autonomous Systems, 34 (2001) 53-63.
Okuno, H.G., Nakadai, K., Lourens, T., Kitano, H.: Sound and Visual Tracking for Humanoid Robot, Applied Intelligence 20 (2004) 253-266.

Parulski, K.A.: Color Filters and Processing Alternatives for one-chip cameras, IEEE Transactions on Electron Devices, 328 (1985) August.

Reece, D.A., Shafer, S.A.: Control of perceptual attention in robot driving, Artificial Intelligence 78, (1995) 397-430.

Sakamoto, Tadashi, et al.: Software pixel interpolation for digital still cameras suitable for a 32bit MCU, IEEE Transactions on Consumer Electronics, 444 (1998) November 1342-1352.

Tang, B. and Lee, K.F.: An efficient color image acquisition system for wireless handheld devices, IEEE ICASSP 2004, 3 (2004) 105-108, Multimedia Architecture Lab Motorola Labs, Schaumburg, Illinois, USA.

Tsai, Y.T. et al.: Optimized Image Processing Algorithms for a Single Sensor Camera, IEEE Pacific Rim Conference on Communications, Computers and Signal Processing, 2 (1997) 1010-1013.

Vijayakumar, S., Conradt J., Shibata, T. e Schaal, S.: Overt Visual Attention for a Humanoid Robot, Proc. International Conference on Intelligence in Robotics and Autonomous Systems, IROS (2001).

Wu, X. et al.: Color Restoration from Digital Camera Data by Pattern Matching, Proceedings of SPIE, 3018 (1997) April 12-17.

Zen, Hidemori, et al.: A New digital signal processor for progressive scan CCD, IEEE Transactions on Consumer Electronics, 442 (1998) May 289295. 